\title{
Comparative glycomic analysis of exosome subpopulations derived from pancreatic cancer cell lines
}

\section{Supporting Information}

Atsushi Matsuda ${ }^{1,2}$, Atsushi Kuno ${ }^{1 *} \ddagger$, Maki Yoshida ${ }^{1} \ddagger$, Takanori Wagatsuma ${ }^{1}$, Takashi Sato $^{1}$, Makoto Miyagishi $^{3}$, Jing Zhao ${ }^{3}$, Makoto Suematsu ${ }^{2}$, Yasuaki Kabe ${ }^{2}$, Hisashi Narimatsu ${ }^{1}$

${ }^{1}$ Biotechnology Research Institute for Drug Discovery, National Institute of Advanced Industrial Science and Technology (AIST), Tsukuba 305-8565, Japan

${ }^{2}$ Department of Biochemistry, Keio University School of Medicine, Tokyo 160-8582, Japan

${ }^{3}$ Biomedical Research Institute, AIST, Tsukuba 305-8566, Japan

*To whom correspondence should be addressed:

Phone/Fax: +81-29-861-3196, E-mail: atsu-kuno@aist.go.jp

\section{CONTENTS}

Figure S1. Lectin blot analysis and lectin array data from Bxpc-3 cells.

Figure S2. Western blot analysis of CD9, CD63, and CD81 in exosome fractions from eight pancreatic cancer cell lines.

Table S1. The abbreviation and carbohydrate specificities of 45 lectins of lectin microarray. 
A
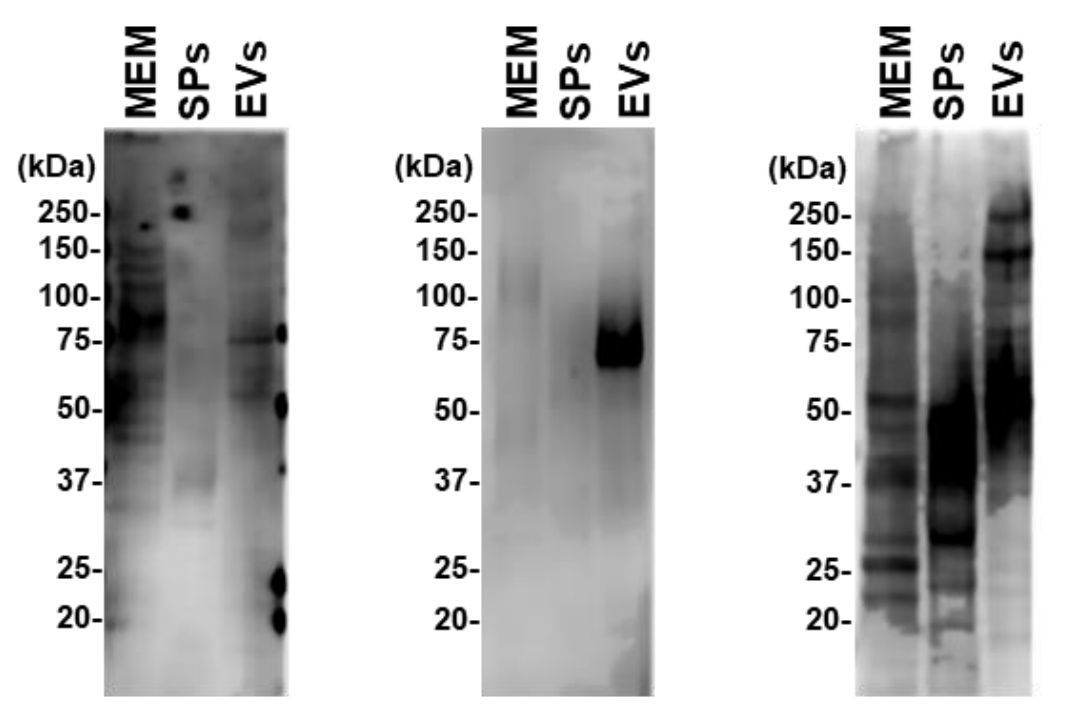

B

HHL
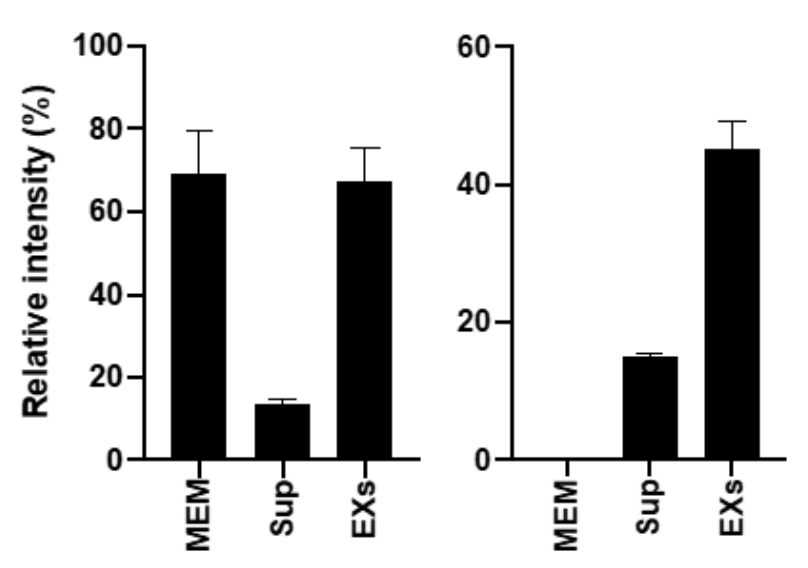

PHA-L

SNA

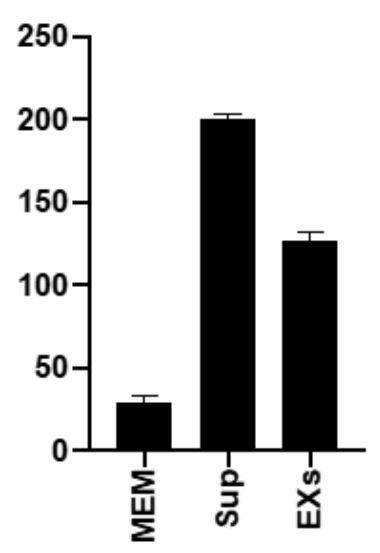

Figure S1. Lectin blot analysis and lectin array data from Bxpc-3 cells.

(A) Lectin blots of membrane, secreted, and exosome surface glycoproteins derived from Bxpc-3 cells. Each protein fraction was loaded at $500 \mathrm{ng} /$ lane. (B) Lectin array of membrane, secreted, and EV glycoproteins derived from Bxpc-3 cells.

MEM; membrane glycoproteins, SPs; secreted glycoproteins. 

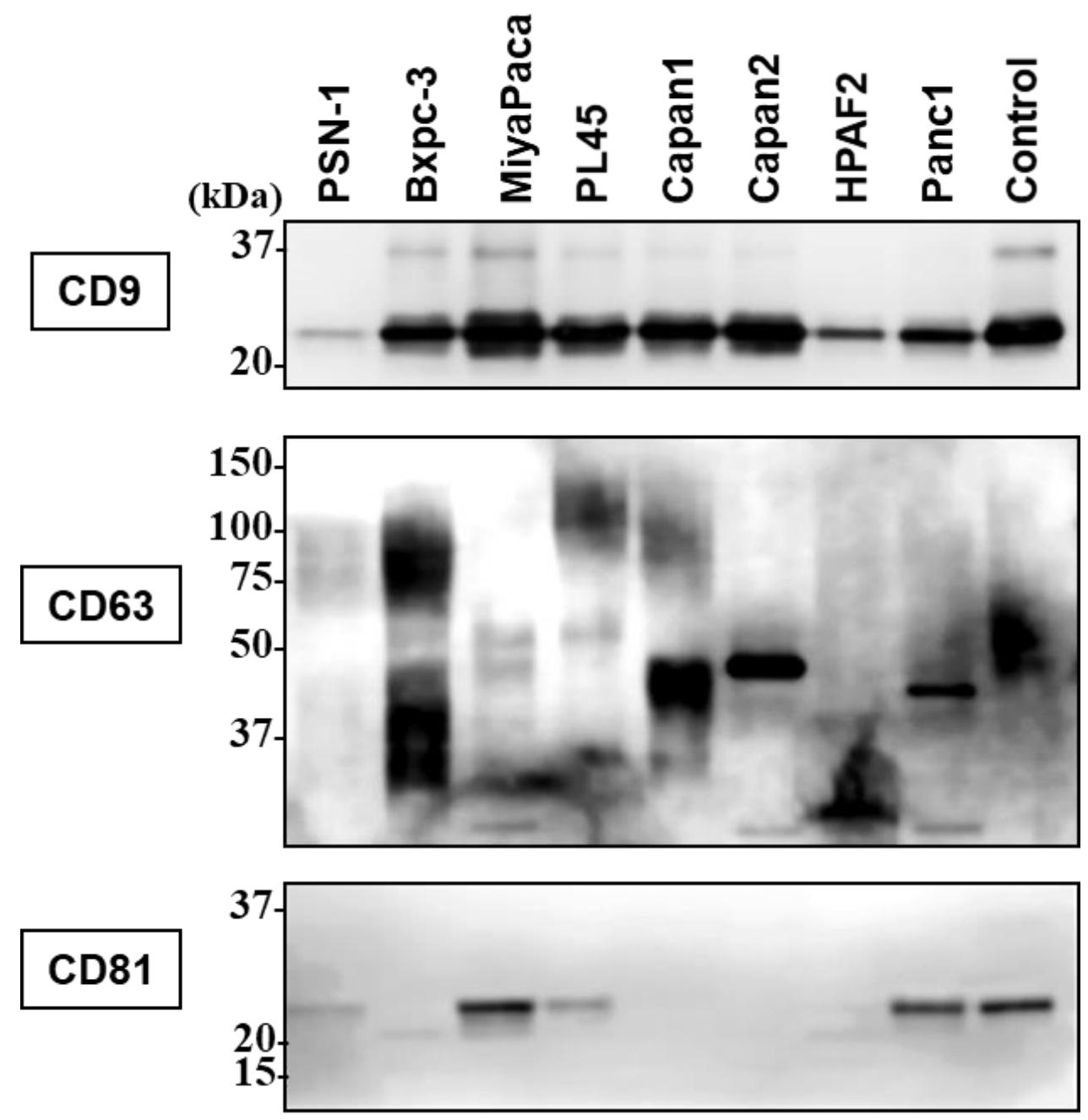

Figure S2. Western blot analysis of CD9, CD63, and CD81 in exosomes from eight pancreatic cancer cell lines. The control represents purified EVs from a colorectal cancer cell line (HCT116) by ultracentrifugation. Each EV sample was loaded at $250 \mathrm{ng} / \mathrm{lane}$ 
Table S1. The abbreviation and carbohydrate specificities of 45 lectins of lectin microarray.

\begin{tabular}{|c|c|c|c|}
\hline & Lectins & Origin & Binding specificity \\
\hline 1 & LTL & Lotus tetragonobus & Fuc $\alpha 1-3$ GlcNAc, Sia-Le ${ }^{x}$ and $L e^{x}$ \\
\hline 2 & PSA & Pisum sativum & Fuc $\alpha 1-6$ GlcNAc and $\alpha-$ Man \\
\hline 3 & LCA & Lens culinaris & Fuc $\alpha 1-6$ GlcNAc and $\alpha$-Man , $\alpha$-Glc \\
\hline 4 & UEA-I & Ulex europaeus & Fuc $\alpha 1-2$ LacNAc \\
\hline 5 & AOL & Aspergillus oryzae & Terminal $\alpha$-Fuc, Sia-Le $e^{x}$ and $L e^{x}$ \\
\hline 6 & AAL & Aleuria aurantia & Terminal $\alpha-F u c$, Sia-Le $e^{x}$ and $L e^{x}$ \\
\hline 7 & MAL & Maackia amurensis & $\mathrm{Sia \alpha 2-3Gal}$ \\
\hline 8 & SNA & Sambucus nigra & Sia 2 -6Gal/GalNAc \\
\hline 9 & SSA & Sambucus sieboldiana & $\mathrm{Sia} \alpha 2-6 \mathrm{Gal} / \mathrm{GalNAc}$ \\
\hline 10 & TJA-I & Trichosanthes japonica & $\operatorname{Sia} \alpha 2-6 \mathrm{Gal} \beta 1-4 \mathrm{GlcNAc} \beta-\mathrm{R}$ \\
\hline 11 & PHA-L & Phaseolus vulgaris & Tri-and tetra-antennary complex oligosaccharides \\
\hline 12 & $\mathrm{ECA}$ & Erythrina cristagalli & $\mathrm{Lac} / \mathrm{LacNAc}$ \\
\hline 13 & RCA120 & Ricinus communis & $\mathrm{Lac} / \mathrm{LacNAc}$ \\
\hline 14 & PHA-E & Phaseolus vulgaris & $\mathrm{NA}_{2}$ and bi secting GlcNAc \\
\hline 15 & DSA & Datura stramonium & $(\mathrm{GlcNAc})_{n}$, polyLacNAc and $\mathrm{L}$ acNAc $\left(\mathrm{NA}_{3}, \mathrm{NA}_{4}\right)$ \\
\hline 16 & GSL-II & Griffonia simplicufolia & Agal actosylated N-glycan \\
\hline 17 & NPA & Narcissus pseudonarcissus & Non-substituted $\alpha 1-6 \mathrm{Man}$ \\
\hline 18 & ConA & Canavalia ensiformis & $\alpha 1-6 \mathrm{Man}$ (inhibited by presence of bisecting GlcNAc) \\
\hline 19 & GNA & Galanthus nivalis & Non-substituted $\alpha 1-6 \mathrm{Man}$ \\
\hline 20 & HHL & Hippeastrum Hybrid & Non-substituted $\alpha 1-6 \mathrm{Man}$ \\
\hline 21 & $\mathrm{ACG}$ & Agrocybe cylindracea & Sia $\alpha 2-3 \mathrm{Gal} \beta 1-4$ GlcNAc \\
\hline 22 & TxLC-I & Tulipa gesneriana & Man 3 core, bi- and tri-antenary complex-type N-Glycan, GalNAc \\
\hline 23 & BPL & Bauhinia purpurea & Gal $\beta 1-3$ GalNAc and $\mathrm{NA}_{3}, \mathrm{NA} 4$ \\
\hline 24 & TJA-II & Trichosanthes japonica & Fuc $\alpha 1-2 \mathrm{Gal}, \beta$-GalNAc $>$ NA3, NA4 \\
\hline 25 & EEL & Euonymus europaeus & Gal $\alpha 1-3[$ Fuc $\alpha 1-2$ Gal $]>$ Gal $\alpha 1-3$ Gal \\
\hline 26 & ABA & Agaricus bisporus & Gal $\beta 1-3$ GalNAc $\alpha-T h r / S e r(T)$ and sialyl-T \\
\hline 27 & LEL & Lycipersicon esculentum & (GlcNAc)n and polyLacNAc \\
\hline 28 & STL & Solamum tuberosum & (GlcNAc)n and polyLacNAc \\
\hline 29 & UDA & Urtica dioica & (GlcNAc)n and polyLacNAc \\
\hline 30 & PWM & Phytolacca americana & (GlcNAc)n and polyLacNAc \\
\hline 31 & Jacalin & Artocarpas integliforia & Gal $\beta 1-3$ GalNAc $\alpha-\operatorname{Thr} / \operatorname{Ser}(\mathrm{T})$ and GalNAc $\alpha-\operatorname{Thr} / \operatorname{Ser}(\mathrm{Tn})$ \\
\hline 32 & PNA & Arachis hypogaea & Gal $\beta 1-3$ GalNAc $\alpha-T h r / \operatorname{Ser}(T)$ \\
\hline 33 & WFA & Wisteria floribunda & Terminal GalNAc (e.g., GalNAc $\beta 1-4$ GlcNAc) \\
\hline 34 & $\mathrm{ACA}$ & Amaranthus caudatus & Gal $\beta 1-3$ GalNAc $\alpha-T h r / \operatorname{Ser}(T)$ \\
\hline 35 & MPA & Maclura pomifera & Gal $\beta 1-3$ GalNAc $\alpha-\operatorname{Thr} / \operatorname{Ser}(\mathrm{T})$ and GalNAc $\alpha-\operatorname{Th} r / \operatorname{Ser}(\mathrm{Tn})$ \\
\hline 36 & HPA & Helix pomatia & Terminal GalNAc \\
\hline 37 & VVA & Vicia villosa & $\alpha-, \beta$-linked terminal GalNAc and GalNAc $\alpha-\operatorname{Thr} / \operatorname{Ser}(\mathrm{Tn})$ \\
\hline 38 & DBA & Dolichos biflorus & GalNAc $\alpha-T h r / \operatorname{Ser}(\mathrm{Tn})$ and GalNAc $\alpha 1-3$ GalNAc \\
\hline 39 & SBA & Glycine max & Terminal GalNAc (especially GalNAc $\alpha 1-3 \mathrm{Gal}$ ) \\
\hline 40 & Calsepa & Calystegia sepium & Man, Maltose \\
\hline 41 & PTL-I & Psophocarpus tetragonolobus & $\alpha$-GalNAc and Gal \\
\hline 42 & MAH & Maackia amurensis & $\operatorname{Sia} \alpha 2-3 \mathrm{Gal} \beta 1-3[\mathrm{Sia} \alpha 2-6 \mathrm{GalNAc}] \alpha-\mathrm{R}$ \\
\hline 43 & WGA & Triticum aestivum & (GlcNAc)n and multivalent Sia \\
\hline 44 & GSL-IA4 & Griffonia Simplicifolia & $\alpha$-GalNAc, GalNAc $\alpha$-Thr/Ser (Tn) \\
\hline 45 & GSL-IB4 & Griffonia Simplicifolia & $\alpha-\mathrm{Gal}$ \\
\hline
\end{tabular}

Data are compiled from the Lectin frontier Database (LfDB; http://riodb.ibase.aist.go.jp/rcmg/glycodb/LectinSerch). 